\title{
Corela
}

Cognition, représentation, langage

HS-25 | 2018

Les procédés implicites dans l'interface sémantiquepragmatique

\section{Les fondements sémantiques de l'implicite argumentatif}

\section{Olga Galatanu}

\section{(2) OpenEdition}

Journals

Édition électronique

URL : http://journals.openedition.org/corela/6577

DOI : $10.4000 /$ corela. 6577

ISSN : 1638-573X

Éditeur

Cercle linguistique du Centre et de l'Ouest - CerLICO

Référence électronique

Olga Galatanu, «Les fondements sémantiques de l'implicite argumentatif », Corela [En ligne], HS-25 |

2018, mis en ligne le 19 juillet 2018, consulté le 12 novembre 2019. URL : http://

journals.openedition.org/corela/6577; DOI : 10.4000/corela.6577

Ce document a été généré automatiquement le 12 novembre 2019.

\section{(c) (1) ()}

Corela - cognition, représentation, langage est mis à disposition selon les termes de la licence

Creative Commons Attribution - Pas d'Utilisation Commerciale - Partage dans les Mêmes Conditions

4.0 International. 


\title{
Les fondements sémantiques de l'implicite argumentatif
}

\author{
Olga Galatanu
}

\section{Introduction}

1 Dans la perspective théorique de la Sémantique des Possibles Argumentatifs (désormais la SPA), à l'interface du sens discursif et de la signification lexicale, nous nous proposons d'analyser trois formes de manifestation discursive de ce que nous pouvons appeler l'implicite argumentatif. Nous posons la définition de ce phénomène sémantico-discursive, comme la reconstruction, dans l'interprétation du sens d'un énoncé ou d'un ensemble d'énoncés, d'un élément signifiant ou d'une configuration d'éléments signifiants relevant de la nature argumentative de la signification d'un mot, présent ou absent de cet énoncé ou de cet ensemble d'énoncés.

2 Cette définition sera reprise, explicitée et illustrée pour chacune de ses parties, dans ce qui suit. Nous nous contenterons, dans cette introduction, de mentionner et d'illustrer les sources empiriques de la conceptualisation que nous proposons d'une forme d'implicite de nature sémantique argumentative.

Dans les recherches en analyse sémantique et en analyse du discours, et en particulier, dans la perspective de l'interface de ces deux disciplines, qui est celle de la SPA (Galatanu 2013, 2016), nous avons identifié de nombreux cas d'implicite de nature argumentative. Des explications comme celles des approches pragmatiques (Grice 1978, KerbratOrecchioni 1986, Moeschler \& Reboul 1998), ou celles des approches discursives s'appuyant sur l'existence d'un "pré-discours » (Paveau 2004), ou de l'inter-discours (Pêcheux 1969, Salazar Orvig 1999, Adam 2006, Paveau 2010, Garric \& Longhi 2013), apportent des éclaircissements importants, sans pourtant faire apparaître nécessairement leurs fondements sémantico-discursifs, notamment en termes de potentialités discursives de la signification lexicale et de potentialités sémantiques du sens discursif produit dans et par les occurrences de parole. 
4 Ainsi, nous allons nous limiter à décrire trois cas d'implicite pour lesquels l'analyse pragmatique et l'analyse discursive fondée sur l'existence d'un pré-discours ne suffiraient pas, à notre avis, à rendre compte du mécanisme linguistique en œuvre ${ }^{1}$, et qui par conséquence, nécessitent une approche complémentaire, en sémantique. Nous allons proposer ensuite un modèle de l'implicite argumentatif, tel qu'il se manifeste dans les trois cas analysés.

5 Après avoir présenté rapidement, dans la première section de l'article, notre approche sémantico-discursive du sens linguistique, la SPA, nous allons avancer, dans la deuxième section, trois hypothèses sur les fondements sémantiques des mécanismes de génération de l'implicite argumentatif. Le modèle du fonctionnement du sens linguistique par l'implicite argumentatif construit sur la base de ces trois hypothèses, lequel permet d'élaborer des solutions élégantes ${ }^{2}$ 'explication de chacun des trois cas identifiés, sera présenté dans la troisième section de l'article. Nous allons investir également le modèle sémantique de l'implicite argumentatif dans une proposition d'analyse de l'insinuation pour chacun des trois cas étudiés.

6 Comme nous l'avons mentionné, nous avons identifié essentiellement trois cas d'implicite argumentatif.

7 A- Il s'agit, pour le premier cas, de la reconstruction d'un faisceau d'enchainements discursifs potentiels, absents de l'énoncé ou de l'ensemble d'énoncés constituant le discours, mais autorisés par la signification d'un mot qui y est mobilisé. Nous retrouvons ici l'idée centrale de L'Argumentation dans la langue (Anscombre \& Ducrot 1983), reprise par de nombreux développements théoriques dans la filiation des sémantiques argumentatives ${ }^{3}$.

8 Dans le cadre de notre réflexion sur une sémantique des possibles argumentatifs, nous avons appelé ailleurs (Galatanu 1999a) cette forme d'argumentation «visée argumentative ». Dans un souci d'adéquation et de cohérence avec l'objet de la présente recherche, nous allons parler d'implicite argumentatif par visée discursive. Nous reprenons ici l'exemple de l'article mentionné, tiré d'un corpus de discours de presse autour d'un événement de 1992 : l'acquittement de la boulangère de Reims qui avait tué d'un coup de fusil l'un des jeunes qui l'agressaient chaque matin dans sa boulangerie.

(1) Depuis des semaines, Ali Rafa [...] et sa bande de potes avaient pris l'habitude [...] d'entrer de force dans la boulangerie de Mme Garnier et de se servir. On peut trouver cette façon de faire très conviviale dans les salons parisiens. Nous, on appelle ça du vol. De la délinquance pure et simple. (Dans Galatanu, 1999a : 50, tiré de Présent, $\mathrm{x}$ ).

9 Une analyse pragmatique qui chercherait à rendre compte de l'implicite, c'est-à-dire, de ce que l'auteur de l'article ne dit pas sur son attitude vis-à-vis de l'acquittement de la boulangère - celle-ci exaspérée par le comportement d'Ali Rafa et de sa «bande de potes », avait tiré avec le fusil de son mari défunt et l'avait tué - devrait présenter plusieurs éléments du contexte :

- l'auteur de l'article est d'extrême droite ;

- il est pour la punition de l'infracteur, puisqu'il ne s'agit pas «d'une façon conviviale d'agir", comme le prétendent les médias de gauche, mais d'un vol qualifié, d'une délinquance pure et simple ;

- il considère que le fait d'avoir été « puni » par la boulangère est juste ;

- il ne peut pas dire qu'il est pour la peine de mort (il a été tué par la boulangère), dans un pays où la peine de mort a été abolie et, dans un Etat de Droit, où il est inacceptable de se 
faire justice soi-même ;

- il enfreint la maxime de quantité et ne dit rien sur la sanction, voire la punition, mais seulement sur le fait que la victime était bien coupable et ceci pour ne pas enfreindre la maxime de qualité (Grice 1957), en mentant sur son point de vue (radical d'extrême droite) sur l'acquittement de la boulangère de Reims.

Une analyse s'appuyant sur l'inter-discours et sur le pré-discours, devrait à son tour faire état aussi des réactions de la presse de gauche qui condamne l'acquittement, de l'interdiscours sur les difficultés des jeunes défavorisés, notamment sur le fait qu'Ali Rafa était jeune et a perdu la vie.

11 Mais, il nous semble difficile d'expliquer la force argumentative de la séquence « Nous, nous appelons ça du vol. De la délinquance pure et simple » et d'interpréter cette séquence comme une argumentation en faveur du pardon de l'acte de la boulangère, si l'on ne procède pas à une analyse sémantique du potentiel argumentatif des mots soulignés.

Ou alors, la solution serait d'ajouter cette analyse à l'analyse pragmatique. Si l'on voulait tester l'interprétation du sens du discours de Présent, dans un cadre intra-discursif (le discours rappelle les faits et finit par attirer l'attention sur "un racisme antiboulangers»), l'attitude de l'auteur de l'article et son plaidoyer en faveur de l'acquittement de la boulangère (plaidoyer dont la seconde partie est implicitée), se trouve justifiée par l'ensemble du discours. L'absence de prise de position par rapport à la sanction /punition du jeune homme, se justifie par le fait que cette position, si elle était explicitée - l'acceptation d'une mise à mort du jeune homme - serait d'une grande violence, d'une violence inacceptable.

B. Le deuxième cas concerne la reconstruction, par le processus d'interprétation du sens discursif, de la signification d'un mot mobilisé par l'énoncé ou l'ensemble d'énoncés formant le discours, pour rendre sémantiquement acceptable ce sens discursif. Nous avons appelé ce mécanisme sémantico-discursif, implicite argumentatif par visée sémantique, comme dans l'exemple (2):

(2) Elle est belle, mais /pourtant elle est intelligente ${ }^{4}$.

L'élément implicite que l'interprétation doit récupérer, pour rendre l'enchaînement discursif sémantiquement acceptable, est l'existence, dans la signification de belle attribuée à une femme, d'un élément associé à la beauté qui serait la bêtise, ou tout au moins la non intelligence. Le sens discursif est entendu comme porteur d'un potentiel d'enrichissement, de régénération, voire même de reconstruction de la signification des mots mobilisés pour le générer.

Une analyse pragmatique devrait rendre compte du contexte ou micro-contexte dans lequel cet énoncé peut être culturellement acceptable et du fait que l'inter-discours et le sens commun le rendent actuellement politiquement et moralement incorrect. Toutefois, pour rendre acceptable le sens discursif, qui pose problème sur le plan sémantique aussi bien que sur le plan culturel, le sujet interprétant devrait accepter :

- qu'il existe/peut exister un lien entre le fait d'être une belle femme (valeur axiologique esthétique positive, entraînant une valeur axiologique hédonique positive : le plaisir à la regarder), et le fait d'être une femme bête (valeur axiologique pragmatique, dans le registre de la compréhension et de la connaissance, négative);

- que ce lien est stable et évident, connu et partagé par la communauté linguistique et 
culturelle, ou tout au moins par les participants à la séquence de communication, à l'échange.

16 Notre modèle de l'implicite argumentatif proposera justement une analyse de la tension entre la signification lexicale, apprise et largement partagée par les membres d'une communauté linguistique, selon le principe de l'obligation formulé par Putnam (1975), et le sens discursif, tension qui se résout par un principe de charité (Galatanu, à paraître), qui agit dans toutes les situations où cette tension se manifeste.

C. Enfin, le troisième cas concerne la reconstruction, par l'interprétation du sens discursif, de la configuration spécifique de la signification d'un mot absent de l'énoncé ou de l'ensemble d'énoncés qui forment le discours, à partir d'éléments de cette signification présents dans cet(ces) énoncé(s). Autrement dit, le mot, avec son signifiant et la configuration d'éléments signifiants qui participent à sa signification "globale », est implicité, mais des éléments signifiants permettent de l'évoquer. Nous proposons d'appeler ce phénomène sémantico-discursif, implicite argumentatif à visée lexicale.

Ce mécanisme, présent par exemple dans les textes de Darwin (voir Meunier et alii 2011) permet que le mot évolution soit si peu présent dans la construction même de la théorie de l'évolution. On le retrouve dans d'autres discours, spécialisés ou professionnels (des pratiques médicales, par exemple), mais aussi dans les interactions verbales interpersonnelles, comme une forme d'évitement de la force menaçante de certains mots ${ }^{5}$

(3) [...]. Le vêtement devient un vecteur de communication du sens propre d'une culture donnée. Elément vestimentaire commun à tous les Premiers Peuples, le mocassin en est probablement l'expression la plus marquante. [...] Ils deviennent la marque d'une tradition culturelle transmise d'une génération à l'autre. Loin d'être statique, le vêtement, comme l'identité, évolue, emprunte et réinterprète. [... ] (Guislaine Lemay, La collection Premiers Peuples, Porter son identité, Musée McCord, 2013).

L'analyse pragmatique de ce texte dans son contexte fait apparaître une stratégie d'évitement du mot patrimoine, considéré par l'auteur probablement comme trop technique ou trop objet de débat sur le périmètre institutionnel du processus de patrimonialisation et donc trop risqué quand il est appliqué à un artefact encore en usage dans les communautés culturelles concernées.

Néanmoins, la récupération de ce mot par l'interprétation est plus difficile à expliquer si l'on ne prend pas en compte le sémantisme des éléments déployés dans le discours qui l'évoquent.

21 Dans ce qui suit, nous allons proposer donc un principe explicatif de ces cas d'implicite, en nous appuyant sur trois hypothèses internes concernant l'interface de la signification lexicale et du sens discursif, entendus comme les deux formes de manifestation du même phénomène langagier, le sens linguistique.

\section{La SPA, une théorie des potentialités discursives de la signification lexicale et des potentialités sémantiques du sens discursif}

22 La SPA s'est donné pour objectif premier, en tant que théorie du sens linguistique, sous ses deux formes de manifestation, la signification des expressions linguistiques, et en 
particulier de la signification lexicale, et le sens discursif, sens produit dans et par chaque occurrence de parole (Galatanu 2013, 2016, à paraître), de décrire la signification linguistique, notamment des mots, comme un processus toujours recommencé de révision de l'univers référentiel que le discours propose dans et par les actes de langage, à travers le sens discursif ${ }^{6}$.

Les conditions qu'une telle théorie doit remplir ont été précisées dès les premiers textes dans le cadre de la $\mathrm{SPA}^{7}$, dans une perspective à la fois argumentative et descriptive (du monde référentiel proposé par le discours):

- être susceptible de rendre compte du potentiel argumentatif, axiologique et descriptif des significations des expressions linguistiques, notamment des mots, id est de l'ancrage culturel et expérientiel de ce potentiel ;

- être susceptible de rendre compte de la partie la plus stable de la signification et de celle la plus instable, ancrée dans les évolutions de la culture d'une communauté linguistique : cette partie aussi est censée être « obligatoirement » connue, donc apprise et partagée par les participants à l'instance de communication, selon le principe de Putnam (Putnam 1975), même si le discours ne cesse d'agir sur elle, pour la régénérer, voire la déconstruire pour la reconstruire.

En tant que sémantique issue de la filiation argumentative ${ }^{8}$ la SPA s'appuie sur un postulat sur la nature argumentative du sens discursif, mais y ajoute, d'une part, un postulat sur l'ancrage dénotatif de la signification, selon lequel, le sens linguistique postule des objets du monde, objets qu'il est susceptible de décrire, à partir de l'expérience intersubjective, collective des locuteurs d'une langue et d'une communauté culturelle. La SPA laisse ainsi une place à l'analyse sémantique de faits discursifs qui relèvent du processus permanent de dénomination".

L'hypothèse externe fondatrice de la $\mathrm{SPA}^{10}$ est que le sens argumentatif, mais également les aspects liés à l'activation d'un lien dénotatif (en particulier dénominatif), s'appuient sur et (ré)inscrivent avec chaque occurrence discursive, un potentiel argumentatif, donc axiologique, et un potentiel descriptif, dans la signification des entités lexicales (Galatanu, 2009b : 56).

Nous développons et argumentons cette hypothèse, qui distingue la SPA des autres approches dans le cadre de ses deux filiations (des sémantiques argumentatives et des sémantiques du stéréotype issues de la réflexion de Putnam 1975), dans plusieurs articles et dans un ouvrage à paraître, que nous avons déjà mentionné.

La signification des mots, telle qu'elle est apprise et acceptée par les locuteurs, conformément au principe de l'obligation de Putnam (Putnam 1975), principe que nous faisons nôtre, a dans le cadre théorique et épistémologique de la SPA, des potentialités discursives, axiologiques, argumentatives et descriptives, qui sont confirmées et régénérées, ou déconstruites et reconstruites par les potentialités sémantiques proposées par le sens discursif ${ }^{11}$. Ces dernières sont fondées sur deux autres principes que nous proposons de prendre en compte, avec celui de l'obligation, pour pouvoir rendre compte de l'interprétation de déploiements discursifs non conformes au protocole sémantique des mots (voir l'exemple (2) ci-dessus) : le principe de coopération, défini et décrit par Grice (Grice 1957) et le principe de charité. Nous reformulons ici ce principe, que nous avons mentionné et/ou défini ou décrit ailleurs (Galatanu 2013, 2017a, b).

L'interprétation du sens linguistique est régie non seulement par le principe d'obligation et un principe de coopération, mais également par un principe de charité, selon lequel les 
sujets interprétants d'un énoncé ou d'un ensemble d'énoncés, acceptent une signification reconstruite, non conforme au protocole sémantique du mot appris et accepté par les locuteurs de la langue, mais conforme au sens discursif, pour rendre celui-ci sémantiquement et culturellement acceptable. Pour élaborer un modèle susceptible de rendre compte des mécanismes de génération du sens discursif, à partir des potentialités discursives, argumentatives et orientées axiologiquement, de la signification lexicale, et de régénération ou reconstruction de cette signification à partir des potentialités sémantiques du sens discursif, y compris des mécanismes de génération de l'implicite argumentatif sous ses trois formes de manifestation identifiées dans ce qui précède ${ }^{12}$, nous avons formulé cinq hypothèses internes. Sans pouvoir détailler, dans l'espace restreint de cet article, ces hypothèses qui fondent le modèle de la représentation du sens linguistique, nous allons présenter la configuration d'associations de mots de la signification lexicale et les liens entre celle-ci et le sens discursif.

La première hypothèse pose l'hétérogénéité de la signification lexicale, qui est appréhendée comme des configurations spécifiques de trois strates à statut différent par le degré différent de stabilité des associations de mots qui les constituent, et par le statut différent des représentations sémantiques de ces mots: le noyau, l'ensemble ouvert de stéréotypes et l'ensemble ouvert des possibles argumentatifs.

Le noyau représente les traits de catégorisation syntaxique et sémantique du mot et les propriétés essentielles à l'identité de l'objet inter-discursif, stabilisé comme un objet sémantique du mot, appris et partagé par la communauté linguistique et culturelle. L'organisation $\mathrm{du}$ noyau est argumentative, par association de représentations sémantiques des mots qui y sont mobilisés, et vectorielle. Ce sont les associations argumentatives les plus stables. Nous reprenons ici le noyau de enseignant, en reprenant une représentation que nous avions proposée ailleurs (Galatanu 2014) et en la remaniant dans le cadre de la version actuelle de la SPA. Nous avons présenté cette représentation du noyau ainsi reconstruit dans une conférence donnée en $2017^{13}$.

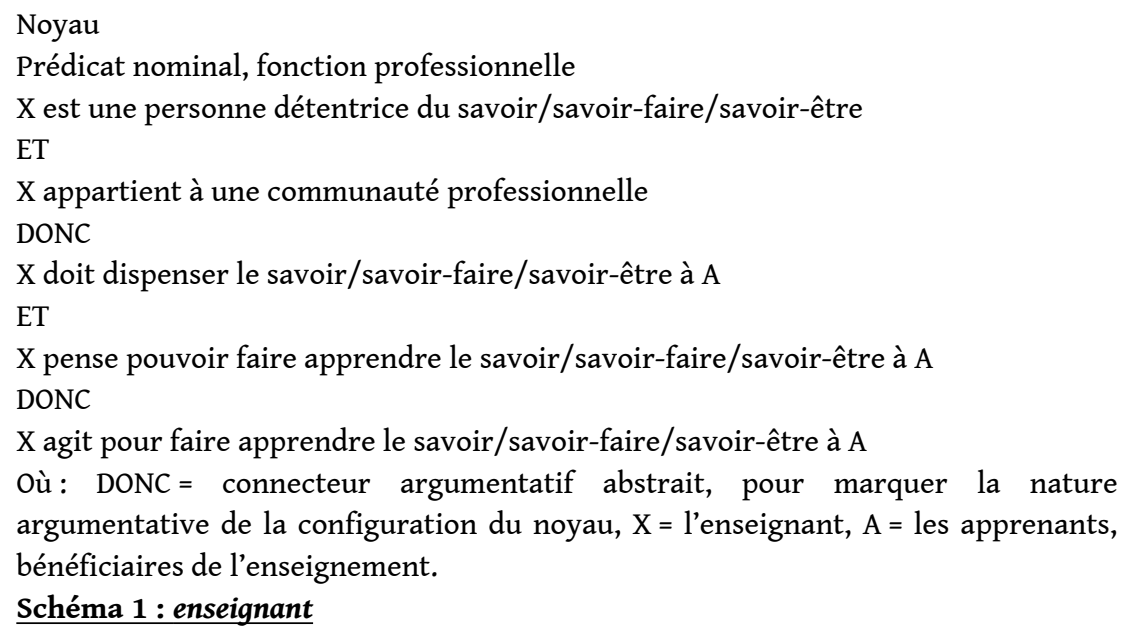

31 Les stéréotypes sont, selon l'hypothèse que nous avions avancée dès les premiers textes de la SPA ${ }^{14}$, puis définis et explicités dans les articles de $2009 \mathrm{~b}$ et 2013), représentent un ensemble ouvert d'associations argumentatives des éléments du noyau avec des représentations conceptuelles et sémantiques portées par des mots du lexique de la langue concernée, représentations ancrées dans les évolutions culturelles, et de ce fait, ${ }^{15}$ cinétiques. 
Parmi les stéréotypes de la signification du mot enseignant, on peut avoir, par exemple: $<\mathrm{X}$ est une personne détentrice du savoir/savoir-faire/savoir-être DONC X a suivi une formation théorique/pratique/professionnelle, $\mathrm{X}$ a des compétences, $\mathrm{X}$ a obtenu des diplômes...>

Les Possibles Argumentatifs (dorénavant PA), qui donnent le nom à la théorie sémantique et au modèle SPA, sont l'une des spécificités de cette théorie et de ce modèle. Ils forment un ensemble ouvert de séquences discursives virtuelles, qui associent le mot à des éléments de ses stéréotypes. Ils sont le produit d'un processus de génération de potentialités discursives du dispositif Noyau-Stéréotypes.

Parmi les PA de enseignant, on peut avoir, par exemple, enseignant donc formation/ compétences/diplômes ....

5 L'hypothèse interne qui nous intéresse tout particulièrement pour notre propos, est celle portant sur l'interface des Possibles Argumentatifs, séquences discursives virtuelles, déjà présentes dans la signification lexicale apprise, d'une part, et les déploiements discursifs de cette signification dans des occurrences de parole, d'autre part. Nous avons ainsi défini ces déploiements discursifs, appelés Déploiements Argumentatifs (DA), comme des séquences discursives, réalisées en contexte, situées, associant le mot concerné avec des éléments de ses stéréotypes. Ces associations peuvent prendre, bien sûr, la forme de séquences argumentatives, avec ou sans connecteur argumentatif explicite, mais elles peuvent être seulement évoquées, par les visées argumentatives des mots mobilisés, ou alors, par la présence des mots associés dans le discours, à des degrés de proximité variable (y compris des collocations dans lesquelles le mot apparaît).

Le sens discursif produit dans l'activité langagière, est appréhendé en SPA comme un déploiement des potentialités discursives de la signification des mots, mais ce déploiement n'est pas simplement une activation des potentialités de sens lexical: ces potentialités sont "contaminées", régénérées, enrichies, par l'environnement sémantique et décohérés ${ }^{16}$, stabilisées le temps de la séquence discursive.

37 Les rapports qui s'établissent entre les PA et les DA représentent non seulement l'espace des mécanismes discursifs de construction du sens discursif, mais également celui de la proposition, par le sens discursif, de potentialités sémantiques, potentialités de signification lexicale, confirmant et régénérant celle apprise et partagée par les locuteurs d'une langue, ou, au contraire, la déconstruisant pour la reconstruire, ne serait-ce que le temps de l'occurrence de parole.

38 C'est dans ce cadre théorique que nous allons avancer nos hypothèses sur l'existence d'une forme d'implicite de nature argumentative et proposer un modèle sémantique de ce phénomène sémantico-discursif.

\section{Les hypothèses d'une sémantique de l'implicite argumentatif}

39 Pour construire un modèle sémantique de la génération de l'implicite argumentatif, tel que nous l'avons défini dans l'introduction à cet article, nous avons avancé trois hypothèses internes concernant les trois cas d'implicite observés ${ }^{17}$, 


\subsection{L'implicite argumentatif par visée discursive}

Hypothèse 1. Nous faisons l'hypothèse que le potentiel argumentatif, axiologique, de la signification lexicale, calculé à partir du dispositif de génération des Possibles Argumentatifs défini par la SPA, représente un faisceau non seulement de potentialités de déploiement discursif, ce qui rejoint la proposition de l'Argumentation dans la Langue (Anscombre \& Ducrot 1983), mais également un faisceau de potentialités d'implicites argumentatifs proposé à l'interprétation de l'énoncé ou de l'ensemble d'énoncés, par le sens discursif. Nous avons appelé ce mécanisme implicite argumentatif par visée discursive.

41 Conformément à cette hypothèse, nous interprétons le sens discursif de séquences comme celles qui sont proposées dans l'exemple (1) - que nous reprenons ici pour les besoins de l'analyse - (4), (5), (6), (7), (8) et (9), comme implicitant des PA de nominaux ou de syntagmes nominaux (prédicat nominal + adjectif qualificatif ${ }^{18}$ ) : vol, délinquance pure et simple, sanction inadaptée, riposte excessive, esclaves, enseignant, professeur stagiaire, communiste, Européenne.

(1) Depuis des semaines, Ali Rafa [...] et sa bande de potes avaient pris l'habitude [...] d'entrer de force dans la boulangerie de Mme Garnier et de se servir. On peut trouver cette façon de faire très conviviale dans les salons parisiens. Nous, on appelle ça du vol. De la délinquance pure et simple. ${ }^{19}$ (Présent, 1992).

(4) Sanction inadaptée

Riposte excessive (Le Monde, 1992, Editorial)

(5) Notre prof, un quinquagénaire tourmenté qui ne s'habillait qu'en noir, exerçait une certaine fascination sur les élèves. "Vous êtes des esclaves ", nous avait-il dit le jour de la rentrée en nous dévisageant un à un. [...] (FRANTEXT: OSMONT Stéphane, Eléments enchaînés, 2012 : 340)

(6) [...] Mais un coup la personne engagée, on lui dit «tu n'es qu'un enseignant » [...]. (Le Nord-Côtier, $\mathrm{n}^{\circ} 12,25$ octobre 2014)

(7) [...] Tu n'es qu'une petite professeure stagiaire, pas une assistante sociale... Une nouvelle fois, des paroles dures... (FRANTEXT : JONQUET Thierry, Ils sont votre épouvante et vous êtes leur crainte, $2006: 155$ )

(8) Qu'est-ce que tu as dans le sang, hein ? Pour un ancien enfant de troupe, c'est pas fort, bon Dieu d'bon Dieu! Je suis bien de l'avis de Buffet... tu n'es qu'un communiste ! (FRANTEXT : GIBEAU Yves, Allons z'enfants, 1952 : 313)

(9) - [...] D'ailleurs, Hermine, tu n'es qu'une Européenne.

- Et toi, qu'es-tu donc?

- Un Russe, Hermine, un Russe qui méprise l'à-peu-près où les forces s'épuisent. J'ai pitié de toi.... (FRANTEXT : CREVEL René, La Mort difficile, 1926:59)

Les exemples (1) et (4), que nous avons étudiés du point de vue de leur visées argumentatives (Galatanu 1999a: 51), proposent des syntagmes nominaux, dans lesquels les prédicats nominaux, à potentiel axiologique monovalent (pour l'exemple (1)) et bivalent (pour l'exemple 4), sont "modifiés $»^{20}$ sémantiquement par des modificateurs sémantiques déréalisants (dorénavant MSD).

Ainsi, pour l'exemple (4), une sanction inadaptée correspond moins au potentiel discursif de sanction (par exemple, justice), y compris au sens juridique du terme (nécessaire, correspondant au délit, juste, etc.). Une sanction, et en particulier, une sanction à la suite d'une décision de justice, dans un Etat de Droit, ne peut être contestée que par le recours à la Justice, à l'institution sociale de la justice. Pour utiliser les termes de l'analyse de Ducrot, 
la sanction inadaptée est donc moins sanction, n'étant plus un rétablissement de la justice, mais une nouvelle transgression de celle-ci.

A son tour, la riposte a un potentiel argumentatif orienté soit vers le pôle positif (éthique-moral ou pragmatique), puisqu'il s'agit d'une réponse à un acte/attitude agressif/agressive, d'une réaction de défense, de contre-attaque, même si, selon un principe d'orientation interne des valeurs modales axiologiques, pour les significations complexes du point de vue de leur modalisation interne (voir Le Thiec 2004), le pôle hédonique-affectif est négatif. Le MSD excessive a, contrairement à l'analyse de Ducrot, non pas une seule fonction, de " déréaliser » le potentiel argumentatif de riposte, mais une double fonction :

- de MSD par rapport à la valeur éthique-morale de la riposte, qui se trouve moins justifiée par son excès,

- de MSR (Modificateur sémantique réalisant) par rapport à la valeur hédonique-affective négative pour le destinataire de la riposte, qui est renforcée par l'excès de la réaction.

Ainsi, sanction inadaptée propose un enchaînement potentiel avec riposte excessive, implicité dans la séquence analysée, et riposte excessive propose à son tour un enchaînement argumentatif, implicité, vers une nouvelle sanction, justifiée par l'excès de la riposte.

L'intérêt de l'exemple (4), comme d'ailleurs de l'exemple (1), réside dans le fait que la reconstruction du potentiel discursif argumentatif et orienté axiologiquement s'appuie sur des propriétés essentielles des prédicats nominaux et/ou de leurs MS (Modificateurs Sémantiques), telles que nous les avons définies dans la configuration argumentative nucléaire de la signification lexicale. Ces exemples font également apparaître le processus de contamination sémantique des propriétés essentielles de ces prédicats et de leurs MS, contamination qui accompagne le processus sémantico-discursif de générationinterprétation de l'implicite argumentatif par visée discursive.

L'énoncé de l'exemple (5) autorise un faisceau de DA à partir des éléments nucléaires aussi bien que des éléments associés aux éléments nucléaires, dans les stéréotypes de esclave : absence de liberté, soumission, travail imposé, souffrance, manque de volonté d'échapper à ce qui est imposé21. La limitation de l'aire possible des PA de esclaves, et donc des DA implicités, est celle d'une « contamination sémantique » avec le potentiel discursif du mot élèves. Le droit de vie et de mort, de vente et d'achat, du propriétaire de l'être humain ayant le statut d'esclave, ne peut pas fonctionner quand l'être humain a le statut d'élève, dans l'institution éducative évoquée par le statut de professeur ${ }^{22}$.

Les énoncés des exemples (6) et (7) sont des structures négatives restrictives, en ne ... que portant sur les identités des destinataires du message que ces énoncés actualisent. Du fait de leur statut de structures restrictives, les énoncés peuvent être considérés comme des "paroles violentes ${ }^{23}$. Ces énoncés autorisent, pour leur interprétation, un faisceau de DA, implicités dans nos exemples, basés sur les stéréotypes des mots enseignant et professeur, orientés vers le pôle axiologique négatif : pragmatique (rôle social, efficacité, salaire). L'exemple (7) renforce cette orientation par deux MSD, qui affaiblissent les stéréotypes orientés vers le pôle axiologique (pragmatique et moral) positif de professeur : petite, MSD générique, et stagiaire, MSD spécifique des prédicats nominaux des métiers et professions.

49 Enfin, les exemples (8) et (9) font apparaitre le mécanisme d'axiologisation négative des stéréotypes de mots axiologiquement bivalents : communisme et Européen, bivalents parce 
que, très ancrés dans les évolutions d'une communauté linguistique et culturelle, ils suivent les dynamiques politiques, idéologiques, des systèmes de valeurs.

Si le mot communiste a connu (et connaît encore) un cinétisme culturel (politique et idéologique) fort, lié à celui du mot communisme, le mot Européen(ne), désignant une appartenance géopolitique en cours de se construire, tout en continuant à désigner une appartenance géographique (à un continent, l'Europe), est encore plus intéressant pour ce qui est du processus d'implicitation de ses PA et de la présence potentielle dans le sens discursif des DA ainsi implicités.

De nombreux stéréotypes culturels sont habituellement associés aux noms désignant les individus appartenant à une identité nationale, à un pays, à une ethnie, à un continent ( les Français, les Japonais, les Africains, les Américains). Ces stéréotypes sont par ailleurs très étudiés par de nombreuses disciplines que nous pouvons regrouper dans les disciplines que Rastier appelle « les sciences de la culture » (Rastier 2002).

Pour Européen, désignant les individus du continent (Europe) ${ }^{24}$, opposé aux noms désignant l'appartenance à un pays/à une nation, le mécanisme sémantico-discursif d'activation ou d'évocation de ses PA est particulièrement complexe. Pour résumer, dans l'espace réduit de cet article, deux cas sont possibles: les noms d'appartenance à un peuple/nation/pays, qu'on lui oppose sont hors les frontières géographiques de l'Europe, ou, au contraire, il s'agit de l'un des pays (l'un des peuples, l'une des nations) de l'Europe.

Dans le premier cas, le déploiement argumentatif implicité évoque un PA basé sur un /des stéréotype(s) antinomique(s) des stéréotypes au nom qu'on lui oppose. Dans notre exemple, ces stéréotypes associés aux Russes sont explicités par des DA du mot Russe: "qui méprise l'à-peu-près où les forces s'épuisent ». Les mécanismes sémantico-discursifs de l'implicite sont, dans ce cas, basés sur l'évocation de stéréotypes de Européenne, non compatibles avec ceux déployés pour Russe, qui peuvent être :

- déjà présents dans la signification du mot Européen, apprise et partagée par la communauté linguistique, susceptibles d'être évoqués et acceptés par l'interprétant, conformément au principe d'obligation de Putnam ;

- proposés à l'interprétation par le locuteur et que l'interprétant peut accepter conformément au principe de charité qui adapte la signification lexicale au sens discursif proposé ;

- proposés à l'interprétation par le locuteur et réfutés par l'interprétant.

\subsection{L'implicite argumentatif par visée sémantique}

Hypothèse 2. Nous faisons l'hypothèse que, si les DA proposés par le sens discursif ne sont pas conformes aux PA de la signification lexicale du mot mobilisé dans l'occurrence de parole, id est à son protocole sémantique, le potentiel argumentatif du sens discursif, à travers les déploiements discursifs qu'il propose, enrichit et reconstruit le potentiel discursif de la signification lexicale, id est ses PA. Les éléments nouveaux, les nouveaux PA que le sens discursif propose à travers ses déploiements argumentatifs non conformes au protocole de la signification lexicale ${ }^{25}$, sont susceptibles d'être reconstruits par les sujets interprétants de l'énoncé produit. Ils représentent ainsi une forme d'implicite argumentatif par visée sémantique, puisqu'ils concernent l'interprétation de la signification lexicale, ne serait-ce que le temps de l'occurrence de parole, conformément au principe de charité. 

cette forme d'implicite argumentatif :

(2) Elle est belle, mais /pourtant elle est intelligente.

(10) C'est un vrai patron d'entreprise, mais il est très sympathique.

(11) Il est intelligent, pourtant il a réussi au CAPES.

(12) Il ne travaille pas beaucoup, il réussira sa vie. ${ }^{26}$

(13) Soyez raisonnables, achetez-vous une voiture de luxe. ${ }^{27}$

列 non conforme au protocole sémantique de ce ces mots et non conformiste culturellement. Pour les exemples (11), (12) et (13), le DA est antinomique par rapport au PA qu'il est censé activer. Il est également antinomique par rapport à la forme converse (qui représente l'exception) de ce PA : <Il est intelligent, pourtant il n'a pas réussi au CAPES>. $\mathrm{Du}$ point de vue axiologique, nous assistons à une flexion de polarité discursive, notamment à une interversion des pôles axiologiques pragmatiques. Les exemples (2) et (10) établissent, par le déploiement discursif proposé, un lien argumentatif entre la beauté des femmes et la non intelligence (2) et entre le statut social et une valeur morale et affective (10). Ces liens ne sont pas prévus dans la signification des mots belle et (non) intelligente, patron d'entreprise et le fait d'être sympathique ou, au contraire, antipathique, et de ce fait, entre des zones d'expérience subjective et intersubjective qui ne sont pas associées par la signification des mots, apprise et partagée par la communauté linguistique. Les sens discursifs pour les cinq énoncés sont acceptables sémantiquement, si les sujets interprétants acceptent de considérer, durant l'occurrence de parole (et, assez souvent, au-delà), que dans la signification de belle, qualité attribuée à une femme, il y a une association avec la bêtise, ou tout au moins avec la non intelligence; dans la signification de patron d'entreprise, il y a un élément antipathique, ou tout au moins non sympathique; dans la signification de réussir au CAPES, la non intelligence; dans celle de travailler beaucoup, un élément échec ou tout au moins non réussite; dans celle de raisonnable, un élément qui serait : faire des dépenses qui dépassent le nécessaire, acheter des objets de luxe.

Ces éléments, que le sens discursif exige pour être acceptable, ne sont pas présents dans les significations apprises et partagées, mais ils sont évoqués par le sens discursif (implicites) comme des conditions de raisonnabilité de la signification lexicale (voir pour les conditions de raisonnabilité des actes de parole: Gordon \& Lakoff 1973). Ils correspondent à des présupposés intervertis axiologiquement, et antinomiques du point de vue de l'orientation argumentative, des mots concernés, non présents dans leur signification, mais imposés par le sens discursif.

\subsection{L'implicite argumentatif par visée lexicale}

58 Hypothèse 3. Nous faisons l'hypothèse que le potentiel argumentatif, axiologique, de la signification lexicale, calculé à partir du dispositif de génération des Possibles Argumentatifs défini par la SPA, peut être déployé dans un énoncé/ensemble d'énoncés sans que le mot ayant ce potentiel soit mobilisé dans cet énoncé/cet ensemble d'énoncés. Ce qui est ainsi implicité, c'est l'entité lexicale, son signifiant et sa signification, qui est évoquée dans le discours par des éléments de sa configuration argumentative, relevant du noyau ou des stéréotypes. Nous appelons cette forme d'implicite, implicite argumentatif à visée lexicale. 
Ce phénomène sémantico-discursif a été mentionné et/ou étudié ailleurs (Galatanu 2003, 2013, à paraître, Didier ${ }^{28}$ 2015) Les exemples (3), que nous reprenons ici, (14), (15) et (16) illustrent la présence de ce phénomène langagier dans des formes de communication de pratiques spécialisées, comme les pratiques de diffusion du patrimoine culturel, ou la pratique médicale (clinique).

(3) [...]. Le vêtement devient un vecteur de communication du sens propre d'une culture donnée. Elément vestimentaire commun à tous les Premiers Peuples, le mocassin en est probablement l'expression la plus marquante. [...] Ils deviennent la marque d'une tradition culturelle transmise d'une génération à l'autre. Loin d'être statique, le vêtement, comme l'identité, évolue, emprunte et réinterprète. [...] (LEMAY Guislaine, La collection Premiers Peuples, Porter son identité, Musée McCord, 2013).

(14) Il y a des toutes petites choses: des nodules au niveau du poumon et du foie ; il faut prendre le relais par un autre traitement et cibler sur ces cellules-là avec une chimiothérapie. (FAINZANG Sylvie $2015: 39$ )

(15) - "Okay", I said. "We have a lot to talk about. If you don't mind, can you tell me what you understand is happening? It's always helpful for me to hear, to make sure I don't leave anything unanswered".

- "Well, I thought I was having a stroke, but I guess... I'm not?"

- "That's right. You aren't having a stroke." I paused, I could see the vastness of the chasm between the life she'd had last week and the one she was about to enter. She and her husband didn't seem ready to hear brain cancer - is anyone? - so I began a couple steps back.

- "The MRI shows a mass in your brain, which is causing your symptoms."

Silence

- "Do you want to see the MRI?"

- "Yes." [...] What's that?" she asked.

- "Could be anything. Maybe an infection, we won't know till after surgery."

My inclination to dodge the question still persisted, to let their obvious worries float in their heads, unpinned.

- "We can't be sure until after surgery", I began, but it looks very much like a brain tumor."

"Is it cancer?"

- "Again, we won't know for certain until it is removed and examined by our pathologists, but if I had to guess, I would say yes."

(KALANITHI Paul (2016), When breath becomes air. What make life worth living in the

face of death, New York, Random House Publishing Group: 92-93)

60 Aucun des trois exemples ne déploie l'ensemble des potentialités de la configuration de la signification des expressions qu'ils évoquent: le patrimoine culturel (exemple (3)) et le cancer (exemples (14) et (15)). Mais les trois exemples déploient soit des mots qui désignent des propriétés essentielles, identificatoires de ces expressions, soit des mots associés à ces propriétés essentielles, nucléaires : identité et transmission d'une génération à l'autre, nodules (au poumon et au foie), a mass (in your brain), chimiothérapie (d'ailleurs appréhendée de plus en plus comme une propriété essentielle dans l'inter-discours qui se construit autour de cette «menace » de la vie).

61 Comme on peut le voir dans ces illustrations, des déploiements discursifs nous conduisent à l'interprétation de la signification lexicale proposée par le sens discursif du patrimoine culturel et du cancer. Ces déploiements représentent, en fait, un potentiel sémantique conforme au potentiel discursif de ces expressions linguistiques. 


\section{Le modèle sémantico-discursif de génération de l'implicite argumentatif}

62 Le modèle de génération de l'implicite argumentatif, élaboré à partir de ces trois hypothèses, se situe à l'interface :

- du potentiel discursif, argumentatif, qui explique la génération du sens discursif ;

- et du potentiel sémantique du sens discursif, qui explique la régénération de la signification lexicale avec chaque occurrence discursive.

63 Le principe explicatif du fonctionnement de l'implicite argumentatif est, comme le principe général du fonctionnement langagier, sous-tendu par ce que nous avons appelé ailleurs l'injonction paradoxale du sens linguistique : la signification lexicale, apprise et partagée par une communauté linguistique et culturelle, contraint la génération du sens (elle déploie ou évoque son potentiel dans les occurrences de parole), et le sens discursif ainsi généré, contraint la régénération, voire même la reconstruction de la signification lexicale (ne serait-ce que le temps de l'occurrence de parole).

64 Le schéma ci-dessous présente synthétiquement le modèle de l'implicite argumentatif, sous-tendu par la tension entre les potentialités discursives de la signification des expressions linguistiques et les potentialités sémantiques du sens discursif.

\begin{tabular}{|c|c|c|c|}
\hline $\begin{array}{l}\text { Explicite/ } \\
\text { Implicite } \\
\text { Types } \\
\text { d'implicite } \\
\text { Argumentatif }\end{array}$ & Explicite & Implicite & $\begin{array}{l}\text { Le processus sémantico- } \\
\text { discursif }\end{array}$ \\
\hline $\begin{array}{l}\text { A visée } \\
\text { discursive }\end{array}$ & $\begin{array}{l}\text { Signification d'une } \\
\text { expression linguistique } \\
\text { (mot ou groupe de } \\
\text { mots) }\end{array}$ & $\begin{array}{lr}\text { Les } & \text { déploiements } \\
\text { argumentatifs r de } \\
\text { l'entité linguistique } \\
\text { mobilisée }\end{array}$ & $\begin{array}{l}\text { Du potentiel discursif de } \\
\text { la signification vers ses } \\
\text { visées argumentatives } \\
\text { pour construire le sens } \\
\text { discursif }\end{array}$ \\
\hline $\begin{array}{l}\text { A visée } \\
\text { sémantique }\end{array}$ & $\begin{array}{l}\text { Sens discursif non } \\
\text { conforme au protocole } \\
\text { sémantique du mot } \\
\text { mobilisé/de } \\
\text { l'expression } \\
\text { linguistique mobilisée }\end{array}$ & $\begin{array}{l}\text { De nouveaux PA de la } \\
\text { signification de } \\
\text { l'expression linguistique } \\
\text { mobilisée, proposés par } \\
\text { le sens discursif }\end{array}$ & $\begin{array}{l}\text { Du sens discursif déployé } \\
\text { vers la reconstruction de } \\
\text { la signification de } \\
\text { l'expression linguistique } \\
\text { mobilisée }\end{array}$ \\
\hline $\begin{array}{ll}\text { A } & \text { visée } \\
\text { lexicale } & \end{array}$ & $\begin{array}{lr}\text { Sens } & \text { discursif } \\
\text { déployant } & \text { la } \\
\text { signification d'un mot } \\
\text { absent de l'énoncé ou } \\
\text { de l'ensemble d'énoncés }\end{array}$ & $\begin{array}{l}\text { L'entité linguistique } \\
\text { dont la signification est } \\
\text { déployée «en son } \\
\text { absence» }\end{array}$ & $\begin{array}{l}\text { Du sens discursif vers } \\
\text { l'entité lexicale évoquée } \\
\text { par ses déploiements } \\
\text { argumentatifs }\end{array}$ \\
\hline
\end{tabular}


Schéma 2 : la construction et l'interprétation de l'implicite argumentatif

- la signification des expressions linguistiques, notamment lexicale, et le sens discursif, du fait de la contrainte exercée par les potentialités discursives de la première sur le second (cas 1);

- le sens discursif et la signification des expressions linguistiques, notamment lexicale, du fait de la contrainte exercée par les potentialités sémantiques du premier sur la seconde (cas 2 et 3 ). Insinuation, id est de ses fondements sémantiques, ce qui ne remet pas en cause l'analyse pragmatique, indispensable pour rendre compte de ce phénomène discursif, en particulier intersubjectif et interactionnel, mais la complète.

par rapport à l'allusion, d'une part et à l'insulte, d'autre part (Grice 1978, Kerbrat-Orecchioni1986, 1998).

me l'a montré Kerbrat-Orecchioni (1998: 43), l'insinuation et l'insulte, se caractérisent par un contenu malveillant, sous-entendu pour la première, explicite pour la seconde. fait apparaître également le fait que le contenu de certaines formes de violence verbale, comme l'insulte, sont susceptibles de générer un conflit et une perte de face ${ }^{30}$ (pour le locuteur qui performe l'acte de parole. Le fait d'évoquer ce contenu négatif, violent, pour la cible/le destinataire de l'acte/du contenu de l'acte, au lieu de le dire explicitement, fait partie, sans aucune surprise, des stratégies d'évitement de cette menace de perte de face (image publique et/ou indépendance) du locuteur (Goffman 1973, Brown \& Levinson 1987).

Chacun des trois cas d'implicite argumentatif peut s'inscrire dans cette stratégie et fonctionner comme une forme d'insinuation, permettant au locuteur de se rétracter devant les conséquences de son acte de parole. Cette interprétation est, évidemment conditionnée par l'orientation négative par rapport à un destinataire ou une autre cible, de son contenu sémantico-discursif ${ }^{31}$.

L'implicite argumentatif par visée discursive peut ainsi représenter une insinuation, si l'entité lexicale (ou le syntagme) n'a pas une orientation axiologique négative pour une cible/un destinataire, devenant ainsi une insulte. Les exemples de (6) à (9), acquièrent une valeur négative pour les destinataires de leurs contenus dans leur environnement sémantico-syntaxique (les structures négatives restrictives) et sémantiques (le MSD petite ), environnement qui produit une flexion de polarité négative. Si insinuation il y a et si on peut la reprocher au locuteur, lui permettant ainsi de se rétracter, elle porte sur l'identité collective d'appartenance de la cible : les enseignants, les professeurs, les Européens.

Corela, HS-25 | 2018 
On peut y répondre: «Tu insinues/ vous insinuez que les enseignants ne sont pas importants dans la société française ? / qu'ils ne méritent pas un bon salaire ? ... ».

Dans le cas de l'implicite argumentatif par visée sémantique, cette interprétation de l'implicite comme une forme d'insinuation portant sur les faits sociaux et sur l'humain, est parfaitement justifiée par la proposition d'une nouvelle signification lexicale, à condition que celle-ci ait subi une flexion de polarité négative: "Tu insinues que les femmes belles ne peuvent pas être intelligentes/que parce qu'elle est belle, elle ne peut pas être intelligente? » pour l'exemple (2), «Tu insinues que ton patron est toujours antipathique?» pour l'exemple (10). Cette forme d'implicite peut même fonctionner comme une insinuation d'un fait axiologiquement négatif, à valeur générique, sur l'humain et le social : «Tu insinues que ce sont ceux qui ne travaillent pas qui réussissent leur vie/que nous, qui avons réussi, nous sommes des feignants?».

6 Enfin, l'implicite argumentatif par visée lexicale, peut aussi fonctionner comme une insinuation, si l'entité lexicale implicitée peut être interprétée comme une insulte, comme c'est le cas de l'exemple de la note 4 que nous reprenons ici :

(16) - Tu ne t'es pas lavé depuis deux jours, tu n'as pas encore nettoyé les vêtements

que je t'avais prêté et tu as laissé traîner sur la table de la cuisine les restes de ton

repas d'hier soir!

- Tu insinues que je suis sale?

- Non, j'attire simplement ton attention sur le fait que je n'aime pas trop comment

tu te comportes comme co-locataire.

Ces exemples ne font qu'ouvrir une piste intéressante pour l'étude des insultes et des insinuations vécues comme des insultes, que nous espérons pouvoir mener à partir de corpus d'interactions verbales authentiques.

\section{Conclusion}

8 Les fondements sémantiques et les mécanismes sémantico-discursifs de l'implicite argumentatif que nous proposons ici permettent une explication cohérente et unitaire des cas identifiés. Pour conclure, nous aimerions souligner le fait que cette explication, ancrée dans l'analyse sémantique des potentialités discursives des significations lexicales, ne vise pas à exclure l'étude du contexte, culturel et situationnel, dans une perspective pragmatique. Au contraire, le choix même d'une forme d'implicite argumentatif nous semble orienter l'analyse vers les racines sociales, culturelles, intersubjectives et subjectives de ce choix. Ce que nous proposons est plutôt une approche complémentaire de faits langagiers traités traditionnellement en pragmatique. Cette approche, centrée sur les fondements sémantiques et les mécanismes sémantico-discursifs de génération du sens discursif et de régénération, voire reconstruction, ou d'évocation des significations des expressions linguistiques, notamment des significations lexicales, est proposée comme un point de départ dans l'analyse des discours et des interactions verbales. Elle autorise un retour vers l'étude du contexte, outillée par ce que le discours construit comme sens en cotexte et en contexte. De ce fait, nous pensons qu'elle peut apporter des éclaircissements nouveaux sur la doxa, sur les idéologies, qui se confrontent et souvent s'affrontent quand le discours laisse de la place et du temps à l'implicite, au non-dit, construit sur le socle sémantique du dit. 


\section{BIBLIOGRAPHIE}

ADAM J-M. (2006), « Intertextualité et interdiscours : filiations et contextualisation de concepts hétérogènes ", TRANEL (Travaux neuchâtelins de linguistique), n 44, p. 3-26.

ANQUETIL S. (2013), Représentation et traitement des actes de langage indirects, Paris, Classiques Garnier, collection Domaines Linguistiques.

ANQUETIL S., BELLACHHAB A. \& GALATANU O. (2015), « La violence verbale au service des idéologies politiques. L'exemple des discours parlementaires sur la burqa », Mémoires de la Société Néophilologique de Helsinki, in PAHTA P., LIIMATAINEN A. \& LARJAVAARA M. (éds.), Tome XCIII, Du malentendu à la violence verbale, p. 263-277. https://tuhat.helsinki.fi/portal/files/49995712/ Dialog3_painoon.pdf

ANSCOMBRE J-C. (dir.) (1995), Théorie des topoï, Paris, Editions Kimé.

ANSCOMBRE J.-C. (2001), « Le rôle du lexique dans la théorie des stéréotypes », Langages $\mathrm{n}^{\circ} 142$, p. $57-76$.

ANSCOMBRE J-C. (2012), « Sens et référence dans la construction d'une sémantique linguistique », Cuadernos de Filologia Francesa, n 23, p. 17-36.

ANSCOMBRE J-C. \& DUCROT O. (1983), L'Argumentation dans la langue, Bruxelles, Mardaga.

BARTMINSKI J. ([2009], 2012), Aspects of Cognitive Ethnolinguistics, Sheffield Oakville, Equinox.

BELLACHHAB A. \& GALATANU O. (2012), « La violence verbale : représentation sémantique, typologie et mécanismes discursifs ", Signes, Discours et Sociétés, $n^{\circ} 9$, La force des mots : les mécanismes sémantiques de production et l'interprétation des actes de parole "menaçants » : http:// www.revue-signes.info/document.php?id=2893.ISSN 1308-8378.

BROWN P. \& LEVINSON S-C. (1987), Politeness: Some Universals in Language Use, Cambridge, Cambridge University Press.

CAREL M. (2011), L'entrelacement argumentative, Paris, Honoré Champion.

COL G. (2017), Modèle instructionnel du rôle des unités linguistiques dans la construction dynamique du sens, Bern, Peter Lang.

DELOOR S. (2012), « Les hypothèses observationnelles en sémantique ; Qui ne glose rien n'a rien », Cuadernos de Filologia Francesa, n 23, p. 37-53.

DIDIER S. (2015), La transmission de l'héritage culturel intangible par la littérature de jeuness. Modélisation sémantico-discursive de "chez soi" dans le conte Mèyènô, (Ponga, 2004), Thèse de doctorat soutenue à l'Université de Nantes et à Macquarie University, Syndeny.

DUCROT O. [1980] (1991), Dire et ne pas dire. Principes de sémantique linguistique, Paris, Hermann.

DUCROT O. (1995), « Les modificateurs sémantiques déréalisants », Journal of Pragmatics, vol. 24, 1-2, p. 145-165.

DUCROT O. \& CAREL M. (1999), « Les propriétés linguistiques du paradoxe : paradoxe et négation ", Langue Française ${ }^{\circ} 123$, p. 27-40. 
FAINZANG S. (2015), La relation médecins-malades : information et mensonge, Paris, Presses universitaires de France.

FRADIN B. (1984), « Anaphorisation et stéréotypes nominaux », Lingua, nº 64, p. 325-369.

GALATANU O. (1999a), « Argumentation et analyse du discours », in GAMBIER Y. \& SUOMELASALMI E. (éds.), Jalons $n^{\circ}$ 2, Université de Turku, Finlande, p. 41-55.

GALATANU O. (1999b), « Le phénomène sémantico-discursif de déconstruction-reconstruction des topoï dans une sémantique argumentative intégrée ", Langue Française, n 123, p. 41-51.

GALATANU O. (2000), « La reconstruction du système de valeurs convoquées et évoquées dans le discours médiatique ", Actes du XXIIe Congrès International de Linguistique et Philologie Romanes, Bruxelles, juillet 1998, Tübigen, Max Niemeyer Verlag, vol. VII, p. 251-258.

GALATANU O. (2004), « La sémantique argumentative intégrée et ses enjeux pour l'analyse linguistique du disccours ", in SALINERO CASCANTE J. \& INAREA LAS HERAS I. (ed.), El Texto como Encrucijada. Estudios Franceses y Francofonos, Volumen II, Lograno, Universidad de la Rioja, p. 203-225.

GALATANU O. (2006), « La dimension axiologique de la dénomination », in RIEGEL M., SCHNEDECKER C., SWIGGERS P \& TAMBA I. (éds.), Aux carrefours du sens. Hommages offerts à Georges Kleiber, Louvain, Peeters, p. 499-510.

GALATANU O. (2009a), « Analyse du Discours dans la perspective de la Sémantique des Possibles Argumentatifs ", in GARRIC N. \& LONGHI J. (éds), L'Analyse linguistique de corpus discursifs : des théories aux pratiques, des pratiques aux théories, Les Cahiers LLL, n³, p. 46-69.

GALATANU O. (2009b), "Semantic and discursive construction of identities. "Europe of knowledge" in the academic discourse", in SUOMELA-SALMI E. \& DERVIN F. (eds), Cross-cultural and Cross-linguistic. Perspectives on Academic Discourse, Amsterdam/Philadelphia, John Benjamins Publishing Company, p. 275-293.

GALATANU O. (2013), « Introduction à l'étude du concept et de la signification lexicale de francophonie. Construction discursive d'un concept, activation d'un lien dénominatif, ou désignation d'un "objet social" ? ", in GALATANU O., COZMA A-M., MARIE V. (dir.), Sens et signification dans les espaces francophones. La construction discursive du concept de francophonie, Bruxelles, Bern, Berlin, Frankfurt am Main, New York, Oxford, Wien, Peter Lang, p. 15-40.

GALATANU O. (2014), « La construction discursive des images et dynamiques identitaires des enseignants et formateurs en FLE/FLS », Signes, Discours et Sociétés, $\mathrm{n}^{\circ} 13$. Sens et identités en construction : dynamiques et représentations : http ;//www.revue-signes.info/document.php ? id $=3525$.ISSN 1308-8378.

GALATANU O. (2016), « “J'ai rencontré une vraie femme, c'est une femme qui nourrit, qui prend soin, qui réconforte, qui écoute, qui accompagne et honore son homme". Les fonctions sémantico-pragmatiques du déterminant nominal vrai », in GAUTIER A., HAVU E. \& VAN RAEMDONCK D. (dir.), DéterminationS, Bruxelles, P.I.E. Peter Lang, p. 145-162.

GALATANU O. (2017a), « Les fondements sémantico-syntaxiques de la poly-fonctionnalité discursive du marqueur illocutionnaire :j'avoue que ", in HADERMANN P., HOUSEN A. \& VAN RAEMDONCK D., La complexité grammaticale dans la structure, l'usage et l'acquisition du langage, Bruxelles, P.I.E. Peter Lang, p. 125-149.

GALATANU O. (2017b), «Quelles ontologies pour une sémantique de l'interaction verbale ? », in HRUBARU F., MOLINE E., VELICU A.-M. (éds), Nouveaux regards sur le sens et la référence. Hommages à Georges Kleiber, Cluj, Echinox, p. 331-359. 
GALATANU O. (à paraître), Sémantique des Possibles Argumentatifs. Génération du sens discursif et (re)construction des significations linguistiques. De la langue au discours. Du discours à la langue, Bruxelles, P.I.E. Peter Lang.

GALATANU O. \& BELLACHHAB A. (2010), «Valeurs modales de l'acte « insulter » et contextes culturels : une approche à l'interface des représentations sémantiques et des représentations culturelles ", Revue de Sémantique et Pragmatique, n 28, p. 123-150.

GARRIC N. \& LONGHI J. (2013), « Atteindre l'interdiscours par la circulation des discours et du sens ", Langage et société, $n^{\circ} 144$, p. 65-83.

GOFFMAN E. (1973), La mise en scène de la vie quotidienne, tomes 1 et 2, Paris, Minuit.

GORDON D. \& LAKOFF G. (1973), « Les postulats de conversation », Langages, n 30, p. 35-56.

GRICE H.P. (1957), « Meaning », The Philosophical Review, n 66, p. 377-388.

GRICE H.P. (1978), “Further notes on logic and conversation”, in COLE P. (ed.), Syntax and Semantics, vol. 9, New York, San Francisco, London, Academic Press, p. 113-127.

GUSDORF G. (1977), La parole, Paris, P.U.F.

KERBRAT-ORECCHIONI C. ([1986]1998), L'implicite, Paris, Armand Colin.

LE THIEC M. (2004), La construction discursive des identités : l'identité des jeunes de la périphérie parisienne dans le discours de la presse française : une étude de cas, Thèse de doctorat soutenue à l'Université de Nantes.

MARANDIN J.-M. (1990), « Le lexique mis à nu par ses célibataires. Stéréotype et théorie du lexique », La Définition, Paris, Larousse, p. 284-291.

MOESCHLER J. \& REBOUL A. (1998), Pragmatique du discours. De l'interprétation de l'énoncé à l'interprétation du discours, Paris, Armand Colin.

MEUNIER J- G., STE MARIE M. \& CHARTIER J-F. (2011), « Did Darwin really talk about Evolution », in AGAZZI E. \& MINAZZI F. (dir.), Evolution and Religion, Florence, Mimesis, p. 127-146.

PAVEAU M.A. (2006), Les prédiscours : sens mémoire, cognition, Paris, Presses Sorbonne Nouvelle.

PAVEAU M.A. (2010), «Interdiscours et intertexte », Annales de l'Université de Franche-Comté, <HAL. 00473985>.

PECHEUX M. (1969), Analyse automatique du discours, Paris, Dunot.

PUTNAM H. (1975), “The meaning of 'meaning”, Philosophical Papers, vol. 2, Cambridge, Cambridge University Press.

RACCAH P. Y. (2014), "Puntos de vista y gestion de los conocimentos", in TORDESILLAS M. \& SUAREZ M.P. (eds), Miradas científicas en torno al lenguaje, Zaragoza, Libros Portico, p. 113-145.

RASTIER F. (2002), « Pluridisciplinarité et sciences de la culture », in RASTIER F. \& BOUQUET S. (dir.), Une introduction aux sciences de la culture, Paris, PUF, p. 1-10.

SALAZAR ORVIG A. (1999), Les mouvements du discours, Paris, L'Harmattan.

VICTORRI B. \& FUCHS C. (1996), La Polysémie, construction dynamique du sens, Paris, Hermès. 


\section{NOTES}

1. D'ailleurs, ce n'est pas l'objectif de ces analyses, centrées sur les liens entre texte et contexte, ou sur la construction des discours et de l'interdiscours.

2. Au sens où l'utilisent les mathématiques : simples et satisfaisantes.

3. Anscombre (dir.) (1995), Ducrot \& Carel (1999), Galatanu (1999a, b, 2004, 2009a), Carel (2011), Raccah (2014).

4. C'est l'un des exemples "fétiches» de la SPA, pour illustrer son principe explicatif du fonctionnement du sens linguistique.

5. On peut imaginer comme illustration, une séquence dans laquelle un locuteur déploie de nombreuses formes de manifestation du qualificatif sale, sans jamais mobiliser ce mot: «Tu ne te laves pas assez, tu portes les mêmes vêtements depuis une semaine, sans les nettoyer, tu laisses traîner les restes de ton repas... ", ou encore, en changeant de cadre temporel : " tu ne t'es pas lavé depuis deux jours, tu n'as pas encore nettoyé les vêtements que tu portes depuis trois jours, tu as laissé traîner sur la table de la cuisine les restes de ton repas d'hier soir ». Ce changement de cadre temporel va nous aider à distinguer, dans ce qui suit, entre « l'accusation d'être sale » et l'insinuation que le destinataire du message est sale (voir la partie 2 de l'article).

6. La SPA s'inscrit ainsi parmi les modèles dynamiques, constructivistes, du sens linguistique. Pour ce paradigme, voir par exemple, Victorri \& Fuchs (1996), Col (2017).

7. Voir à ce sujet Galatanu, 1999b, 2004.

8. Il s'agit, en particulier, des travaux de Ducrot (1980), Anscombre \& Ducrot (1983), Anscombre (dir.) (1995), Ducrot (1995), Ducrot \& Carel (1999), Galatanu (1999a, b, 2000).

9. Voir Galatanu (2006, à paraître).

10. Elle correspond à ce que Ducrot (1980), appelle les hypothèses externes et Anscombre (2012), Deloor (2012) appellent les hypothèses observationnelles d'une théorie sémantique scientifique.

11. Une discussion plus détaillée de cette spécificité de la SPA est proposée dans l'ouvrage mentionné.

12. Nous ne prétendons pas épuiser le potentiel de génération d'implicites argumentatifs de l'interface signification lexicale-sens discursif. Nous nous contentons ici de proposer, illustrer et argumenter les trois cas déjà identifiés.

13. Galatanu, O. (2017), « De la producción de saberes de acción a la producción de sí mismo. Procesos cognitivos y discursivos, afectos y valores ", conférence plénière au 1er Colloque International Practica y teoría en la formación de los profesores y en la enseñanza de lenguas extranjeras, Bogota, 4-6 octobre 2017.

14. Galatanu $(2000,2004)$.

15. Plusieurs spécificités de la SPA distinguent les stéréotypes qu'elle définit de ceux définis par d'autres sémantiques du stéréotype, dans la filiation de la réflexion de Putnam (Fradin 1984, Marandin 1990, Anscombre 2001, Bartminski 2009) : le fait qu'il s'agisse d'un ensemble ouvert, leur association avec un ou plusieurs éléments du noyau, le fait qu'ils peuvent être antinomiques, comme la faiblesse et la douceur, mais aussi la dureté, la capacité de supporter la douleur, associées à la féminité. Ces spécificités qui apparaissent dans les textes qui fondent la SPA, mais aussi dans de nombreuses recherches sur corpus discursifs ou des données élicitées, dans ce cadre théorique, sont détaillées et illustrées dans Galatanu, à paraître.

16. Au sens que donne à ce concept la physique quantique.

17. Ceci revient à dire, que nous partons des trois hypothèses observationnelles (externes) sur l'existence d'une forme d'implicite dans les séquences discursives étudiées.

18. Ces adjectifs qualificatifs sont appréhendés et décrits par Ducrot (1995) comme des modificateurs sémantiques (MS) « réalisants» (MSR), qui renforcent le degré d'application du 
sens argumentatif du nominal, ou du verbe, ou "déréalisants" (MSD), qui affaiblissent le potentiel argumentatif des nominaux ou des prédicats verbaux.

19. Cet exemple et l'exemple 4 sont proposés dans Galatanu (1999a).

20. Le terme de Modificateur Sémantique nous pose problème puisque, selon la définition de Ducrot, il ne s'agit pas de modifier la signification du mot, mais de renforcer ou d'affaiblir son potentiel argumentatif. Toutefois, dans nos exemples, les MS appliqués à des bivalents, agissent sur ce potentiel axiologique et argumentatif bivalent, par une détermination de l'orientation axiologique vers le pôle négatif ou positif. Le terme de modificateur prend tout son sens ici.

21. Tout au moins pour ce qui est de l'attitude de soumission qu'un esclave doit afficher pour survivre. Il nous semble que c'est prioritairement cette attitude qui est activée dans la métaphore proposée dans l'exemple (5).

22. Nous faisons l'hypothèse que ce point de vue sur la limitation discursive du déploiement du potentiel descriptif de la signification lexicale, par contamination avec l'environnement sémantique peut se trouver à la base d'un principe explicatif du processus de métaphorisation. Nous avons exposé un début de ce principe explicatif dans le cadre de la SPA, dans une conférence donnée à Milan: Galatanu O. (2016), «Expressions imagées et axiologisation discursive pour dire la f/Francophonie », Università degli studi di Milano, 14 mars 2016.

23. Sur la définition et l'analyse que nous proposons de la parole violente: voir Galatanu \& Bellachhab (2010), Bellachhab \& Galatanu (2012), Anquetil, Bellachhab \& Galatanu (2015).

24. L'ouvrage dont l'exemple est tiré est de 1926. Dans le contexte actuel être Européen renvoie également à la prise de position par rapport à la construction européenne, ce stéréotype nouveau confirmant le cinétisme culturel et linguistique du mot.

25. Nous pouvons dire aussi que ces éléments sont «non conformistes » culturellement, comme l'association de la beauté de la femme avec la non intelligence. En français, il existe un dicton, qui évoque le topos mobilisé dans notre exemple, « Sois belle et tais-toi !", mais cette association est devenue politiquement et moralement incorrecte dans l'inter-discours construit dans la société contemporaine.

26. Les exemples (2), (10), (11), (12) sont des exemples construits.

27. L'exemple (13) est une publicité des années 1990.

28. Didier parle de non-occurrence (du mot) home/chez soi, dans le conte kanak de la Nouvelle Calédonie, Mèyyènô (Ponga, 2004).

29. Galatanu \& Bellachhab (2010), Bellachhab \& Galatanu (2012), Anquetil, Bellachhab \& Galatanu (2015).

30. Voir à ce sujet Goffman $(1973,1974)$, Gusdorf (1977).

31. Le sémantisme du contenu propositionnel de l'acte de parole participe à la construction de la force illocutionnaire de l'acte de parole. Voir à ce sujet Anquetil (2013).

\section{RÉSUMÉS}

L'article propose l'analyse de trois formes de manifestation de ce que nous avons appelé l'implicite argumentatif: à visée discursive, à visée sémantique et à visée lexicale. Dans la perspective théorique de la Sémantique des Possibles Argumentatifs, nous avons défini ce phénomène sémantico-discursif, comme la reconstruction, dans l'interprétation du sens d'un énoncé ou d'un ensemble d'énoncés, d'un élément signifiant ou d'une configuration d'éléments 
signifiants relevant de la nature argumentative de la signification d'un mot, présent ou absent de cet énoncé ou de cet ensemble d'énoncés. L'article défend, illustre et argumente les fondements sémantiques de ces trois types d'implicite argumentatif, ancrés dans le potentiel discursif de la signification des mots et dans le potentiel du sens produit dans les instances de parole, de proposer des significations lexicales régénérées.

This paper proposes the analysis of three manifestation forms of a discursive phenomenon which we name argumentative implicit: discursive aimed, semantic aimed and lexical aimed. In the theoretical perspective of Semantics of Argumentative Possibilities, we define this semanticdiscursive phenomenon as reconstruction of a significant element within the argumentative nature of the word meaning. The word may be present or absent in the discourse. We defend, illustrate and argue the semantic foundations of these three types of argumentative implicit, which are based on the discursive potential of words meaning or the semantic potential of the discourse sense to propose lexical regenerated meanings.

\section{INDEX}

Mots-clés : Sémantique des Possibles Argumentatifs, implicite argumentatif, visée discursive, visée sémantique, visée lexicale

Keywords : Semantics of Argumentative Possibilities, argumentative implicit, discursive aimed, semantic aimed, lexical aimed.

\section{AUTEUR}

OLGA GALATANU

CoDiRe, EA 4643, Université de Nantes 